\title{
Revisão Sistemática para estudo de Interação Criança- Computador associada a RA e Jogos Digitais
}

\author{
Fabio Alexandre Caravieri Modesto ${ }^{1,2}$, Pollyana Notargiacomo Mustaro \\ ${ }^{1}$ Instituto Federal de Educação Ciência e Tecnologia de São Paulo (IFSP) - Campus \\ Salto \\ Rua Rio Branco, 1780 - 13.320-271 - Salto - SP - Brazil \\ ${ }^{2}$ Universidade Presbiteriana Mackenzie (UPM) \\ Rua da Consolação, 930 - 01.302-907 - São Paulo, SP - Brazil
}

\begin{abstract}
fabio@fabiomodesto.pro.br, pollyana.mustaro@mackenzie.br
Abstract. Considering the use of computing devices (mobile or not) by the children, as well as the emergence of the relevant studies related to interfaces for children (Child Computer Interaction-CCI), it was attempted to, through the realization of a systematic review, analyze concerning the development of digital games with Augmented Reality (AR) research. As a result it was obtained a list of 17 articles, and of these only ten met the proposed questions. Among the results, it was found that the applications are recent and directed to the educational process, constituting a gap in literature digital games strictly for the children's entertainment. It was also possible to map the base of scientific articles that explores the combination of ICC, RA and digital games as well as a specific event that discussions in this regard are undertaken.
\end{abstract}

Resumo. Tendo em vista o uso de dispositivos computacionais (móveis ou não) por parte das crianças, bem como o surgimento de estudos pertinentes à ao uso de interfaces para crianças (Interação Criança Computador - ICC), buscou-se, por meio da realização de uma revisão sistemática, analisar as investigações concernentes ao desenvolvimento de jogos digitais com Realidade Aumentada (RA). Como resultado obteve-se uma lista de 17 artigos, sendo que destes apenas dez satisfaziam os questionamentos propostos. Dentre os resultados, identificou-se que as aplicações são recentes e voltadas aos processos educacionais, constituindo uma lacuna na literatura os jogos digitais estritamente para o entretenimento infantil. Também foi possível mapear a base de artigos científicos que explora a combinação de ICC, RA e jogos digitais, bem como um evento específico em que discussões a esse respeito são empreendidas.

\section{Introdução}

Vivencia-se um período de mudança na estrutura comunicacional da sociedade, que abarca também a educação, de modo geral. Esta transformação procede, dentre os outros fatores, das novas tecnologias, sendo que elas podem ser consideradas como agente mediador de processos de construção de conhecimento. 
A utilização pedagógica do computador, da Internet e da novas mídias como veículo de promoção da aprendizagem constitui uma temática que busca encontrar caminhos que ampliem a qualidade do processo de ensino e aquisição do conhecimento. Dentre as propostas contemporâneas, pode-se destacar o Construcionismo. Este foi proposto na década de 80 por Seymour Papert (2008) e pauta-se na premissa de que ao invés do computador ser utilizado como um veículo de instrução (instrucionismo) este deve constitui uma ferramenta, ou seja, mediar o desenvolvimento de soluções, facilitando a descrição, a reflexão e a depuração das ideias. Assim, também se requer uma alteração de paradigma no que se refere ao professor, cuja responsabilidade voltase para a contribuição na formação de pessoas capazes de criar, pensar e construir. Portanto, é importante que o uso pedagógico do computador seja planejado de forma a considerar o aluno como centro do processo.

Crianças utilizam e possuem anseios, em relação à tecnologia e aos computadores, diferentes dos adultos. Contextualizando tal questão na área de IHC (Interface HumanoComputador) se percebeu a necessidade de direcionar pesquisas para o estudo de interfaces para as crianças (Interação Criança Computador - ICC). Ainda neste contexto, Jogos em Realidade Aumentada (RA) proporcionam uma interface mais amigável por trazer o mundo computacional para o espaço do usuário, instituindo uma interação mais livre (se comparada aos consoles cuja interface relaciona-se com o hardware e dispositivos de cada um dos fabricantes). Assim, a RA institui novas possibilidades para a produção de jogos, em contraste aos controles de jogos tradicionais e também ao uso de dispositivos ubíquos.

Da mesma forma, para uma educação efetiva na atualidade se faz necessário associar ao processo de aprendizagem elementos que estabeleçam aderência entre os estudantes e o conteúdo de uma forma motivadora. A associação de recursos tecnológicos com métodos pedagógicos pode ser uma forma de se obter este objetivo. Tecnologias como Realidade Virtual (RV), Realidade Aumentada (RA) e Jogos Digitais com conteúdos pedagógicos, adaptados adequadamente, poderiam ser utilizadas, como forma de motivação para os alunos, considerando a metáfora lúdica que estes propiciam [Barbatsis et al. 2011].

O uso deste tipo tecnologia na educação pode explorar estímulos estereoscópicos, que têm como objetivo gerar dois fatores: a absorção e a presença emocional dos estudantes diante dos conteúdos a serem aprendidos. Por exemplo, uma aula de história da Roma Antiga poderia fazer uso de uma simulação de construções e cidades, sendo que os estudantes poderiam caminhar pelos ambientes e "vivenciar" como estes eram, ampliando as possibilidades anteriores (visualização por fotos de livros ou museu) [Harada, Nosu e Okude 1999]. Por outro lado, seria possível gerar ambientes cujos acessos são dificultados por questões naturais ou mesmo inexistentes: interior de cavernas, oceanos ou um planeta.

A partir destes pressupostos, buscou-se realizar uma revisão sistemática que estabelece a interseção entre ICC, RA e jogos educacionais. Para isso, o presente trabalho encontra-se estruturado conforme os seguintes itens: Fundamentos Teóricos (discorre sobre os conceitos pertinentes à investigação); Planejamento e Realização da Revisão Sistemática (trata da estruturação e execução da revisão sistemática), Resultados Obtidos (organiza os dados coletados); Análise das Pesquisas que combinam 
ICC e RA para jogos educacionais (em que se discutem os artigos que satisfizeram os critérios de inclusão na investigação); e Considerações Finais (em que se destacam as conclusões do estudo e as investigações a serem realizadas em seguida).

\section{Fundamentos Teóricos}

Tendo em vista a inter-relação das três áreas (RA, ICC e Jogos Digitais Educacionais) na presente revisão sistemática, abaixo se encontram dispostos os conceitos pertinentes às mesmas.

\subsection{Realidade Aumentada}

A Realidade Aumentada (RA) possibilita o amalgama de imagens sintéticas, que podem ser em Segunda Dimensão (2D) e/ou Terceira Dimensão (3D), geradas por computador, com imagens do mundo real (físico). Sua aplicação ocorre em diversas áreas, como por exemplo: educação, engenharia, medicina e no entretenimento, onde se pode observar especial destaque no desenvolvimento de jogos eletrônicos. A RA estabelece uma nova relação na interface humano-computador, pois estende a forma de interpretar as interfaces gráficas tradicionais (Graphic User Interface - GUI) [Tori et. al. 2011].

A RA transporta os jogos do ambiente computacional até o espaço do usuário, viabilizando uma interface amigável, fornecendo uma experiência de ação natural e agradável, que pode ser visualizada por um Head-Mounted Display (HMD) ou indiretamente por um monitor de vídeo e utilizando as mãos, na aplicação de RA. Assim, os usuários podem manipular os objetos do mundo real e virtual, no ambiente gerado, sem a necessidade do uso de equipamentos como joysticks (controles), teclados ou mouse [Kirner et. al. 2006].

\subsection{Interface Criança Computador}

As pesquisas de Interface Humano-Computador (IHC) foram originadas em estudos voltados à ergometria e ao fornecimento de orientação em sistemas computacionais, sendo que atualmente as investigações têm se voltado à usabilidade, à ludicidade, à produtividade, à comunicação e controle. A Interação Criança Computador (ICC) constitui uma área de pesquisa recente no âmbito da IHC, sendo que se encontra em crescimento devido, principalmente, as suas potencialidades de utilização na área de educação. Destaca-se que ICC tem constituído tema em eventos relacionados à IHC, possuindo, inclusive, um evento específico (Interface Design and Children), sendo que tal abordagem se reflete no crescimento de pesquisas e publicações na área, expresso na Figura 1 [Read e Bekker 2011]. 


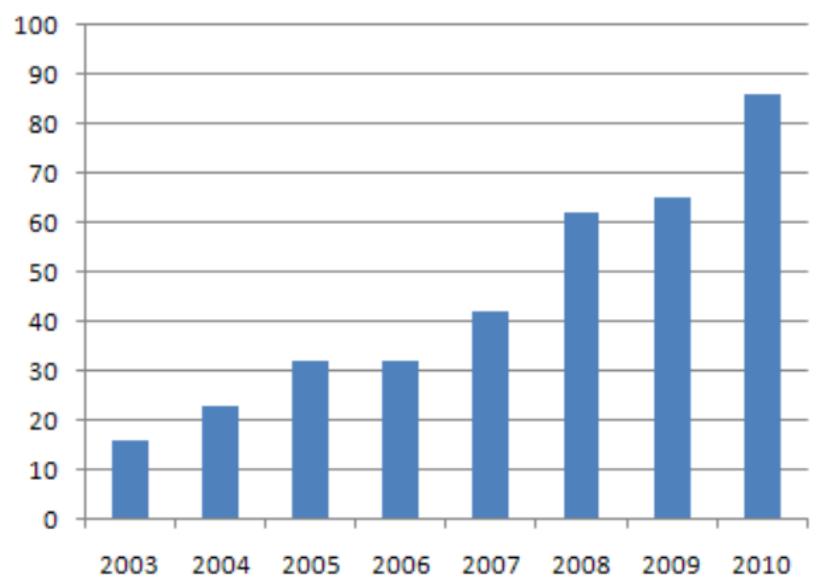

Figura 1. Números de artigos publicados em eventos de IHC que possuem "criança" como conteúdo (Read e Bekker 2011, p. 2)

Segundo Read e Bekker (2011) a ICC pode ser caracterizada como o segmento de IHC em que os humanos são crianças, sendo que são consideradas as diferenças destas no que se refere à faixa etária e às necessidades de interação. Portanto, considerase que crianças têm atividades, comportamentos e usabilidade em relação ao computador distintas dos adultos.Quando se pensa em crianças usando tecnologias podem-se especificar, basicamente, dois cenários: 1) casa ou 2) escola. Como socialmente crianças passam mais tempo na escola que em casa, socialmente existe uma partilha entre estes dois cenários, sendo que o contexto de maior utilização é em casa. $\mathrm{O}$ paralelo entre criança e interação está mais relacionado à natureza de entretenimento e aprendizado do que de trabalho. Uma estratégia de interação eficaz, considerando uma abordagem ICC, será entendida não como o computador é alimentado por trabalhos do mundo real, mas como ele é alimentado pelo mundo dos jogos e da educação.

\subsection{Jogos em Processos Educacionais}

Para Vygotsky (2001), a brincadeira se configura como um lócus diferenciado e privilegiado para a aprendizagem infantil. Isso ocorre porque esta (brincadeira) encerra uma estruturação básica para a modificação de necessidades e consciência. Ao brincar as crianças questionam e enfrentam desafios que ultrapassam seu cotidiano, instituindo o levantamento de hipóteses como veículo para a compreensão dos problemas propostos a elas e pela na qual interagem.

Assim, torna-se relevante investigar as necessidades, motivações e tendências que as crianças manifestam e como se satisfazem nos jogos, a fim de compreender os avanços nos diferentes estágios de seu desenvolvimento. Ao caracterizar o brincar da criança como imaginação em ação se faz com que o brinquedo traduza uma situação imaginária, trazendo em sua concepção uma regra relacionada com o que está sendo representado. Assim, quando a criança brinca de médico, busca agir de modo muito próximo daquele que ela observou nos médicos do contexto real. A criança cria e se submete às regras do jogo ao representar diferentes papéis [Vygotsky 2001].

Devido ao enfoque cientifico que se concedeu às pesquisas em RA, e sua aplicação nas mais distintas áreas, especialmente no desenvolvimento de jogos eletrônicos por apresentar uma interface gráfica que transcende as interfaces 
tradicionais, convém conhecer o segmento das pesquisas sobre esta tecnologia no escopo da área educacional para crianças.

\section{Planejamento e Realização da Revisão Sistemática}

A partir dos pressupostos anteriores, a pesquisa se concentrou em artigos que abordavam jogos digitais com RA, existentes nas seguintes bases de conhecimento: IEEE, ACM e Scorpus. Para obtenção das publicações foi utilizado o seguinte argumento de pesquisa: "child computer interaction" AND "augmented reality" AND "games".

Tal abordagem foi adotada para responder aos seguintes questionamentos (principais - QP e secundários - QS):

- QP1: As pesquisas que combinam ICC e RA a jogos digitais são voltadas ao entretenimento per se ou destinam-se a abordagens educacionais?

- QP2: Há uma diferença numérica em relação à ocorrência de artigos listados (publicados em eventos e/ou periódicos) nas bases científicas investigadas em relação aos três elementos estudados (ICC, RA e jogos digitais)?

- QS1: Seria possível delimitar em que período se iniciou as pesquisas envolvendo ICC combinada à RA e jogos digitais?

- QS2: Existem eventos e/ou periódicos específicos que exploram a relação ICC, RA e jogos digitais?

A busca retornou apenas 17 publicações, todas estas disponibilizadas na base da ACM. A seleção das publicações se deu observando, em primeiro lugar, o título e abstract; quando estes não permitiam uma conclusão clara do objeto do artigo, o mesmo era lido na íntegra para se evidenciar o conteúdo. Foram definidos como critérios de inclusão se artigo tratava de Jogos Digitais com RA e se focavam Interação Criança Computador. Os critérios de exclusão foram concernentes a todos os artigos que não satisfaziam as propriedades descritas anteriormente.

\section{Análise das Pesquisas que combinam ICC e RA para Jogos Digitais Educacionais}

O critério de inclusão de trabalhos na presente revisão sistemática se baseou na relação dos temas ICC, RA e jogos digitais. Neste sentido, buscou-se verificar se a RA foi aplicada como interface entre a criança e o computador de forma que ela apresentasse uma metáfora de interação amigável, lúdica, tangível e natural, evitando o elenco dos dispositivos convencionais de interação (mouse e joystick) e, assim, proporcionando uma interação dinâmica e obtenção de feedback motivacional positivo por parte das crianças que participaram das pesquisas levantadas.

Em relação aos artigos excluídos da revisão sistemática (sete no total), destacase que os problemas encontrados, que constituíram fatores de eliminação da amostra, referiam-se a problemas conceituais em relação à RA, direcionamento para interação social (sem descrição ou relevância da tecnologia empregada), inclusão social e discussão de questões de ICC sem relação com os demais temas (RA e jogos digitais). 
Assim, dos 17 artigos encontrados inicialmente foram analisados dez, sendo que os mesmos encontram-se listados na Tabela 1, abaixo:

\begin{tabular}{|c|c|c|}
\hline Título / Autores do Artigo & Periódico ou Evento (Publicação) & Ano \\
\hline $\begin{array}{l}\text { aMAZEd Designing an Affective Social Game for } \\
\text { Children [Mahmud et al. 2007] }\end{array}$ & $\begin{array}{l}\text { Proceedings of the } 6^{\text {th }} \text { International Conference on } \\
\text { Interaction Design and Children (IDC '07) }\end{array}$ & 2007 \\
\hline $\begin{array}{l}\text { Editorial the evolving field of tangible interaction } \\
\text { for children [Zaman et al. 2012] }\end{array}$ & Personal Ubiquitous Comput. & 2012 \\
\hline $\begin{array}{l}\text { Bringing Tabletop Technologies to Kindergarten } \\
\text { Children [Marco et al. 2009] }\end{array}$ & $\begin{array}{l}\text { Proceedings of the } 23^{\text {rd }} \text { British HCI Group Annual } \\
\text { Conference on People and Computers: Celebrating } \\
\text { People and Technology (BCS-HCI '09) }\end{array}$ & 2009 \\
\hline $\begin{array}{l}\text { Children and intuitiveness of interaction: a study } \\
\text { on gesture-based interaction with augmented } \\
\text { reality [Lagerstam, Olsson e Harviainen 2012] }\end{array}$ & $\begin{array}{l}\text { Proceeding of the } 16^{\text {th }} \text { International Academic } \\
\text { MindTrek Conference (MindTrek '12) }\end{array}$ & 2012 \\
\hline $\begin{array}{l}\text { HeartBeat: An Outdoor Pervasive Game for } \\
\text { Children [Magielse e Markopoulos 2009] }\end{array}$ & $\begin{array}{l}\text { Proceedings of the SIGCHI Conference on Human } \\
\text { Factors in Computing Systems (CHI '09) }\end{array}$ & 2009 \\
\hline $\begin{array}{l}\text { Incorporating Peephole Interactions into } \\
\text { Children's Second Language Learning Activities } \\
\text { on Mobile Devices [McNally 2014] }\end{array}$ & $\begin{array}{l}\text { Proceedings of the } 2014 \text { Conference on Interaction } \\
\text { Design and Children (IDC '14) }\end{array}$ & 2014 \\
\hline $\begin{array}{l}\text { Investigating the Educational Effectiveness of } \\
\text { Multiplayer Online Games for Children [Garzotto } \\
\text { 2007] }\end{array}$ & $\begin{array}{l}\text { Proceedings of the } 6^{\text {th }} \text { International Conference on } \\
\text { Interaction Design and Children (IDC ' } 07 \text { ) }\end{array}$ & 2007 \\
\hline $\begin{array}{l}\text { MOSOCO: A Mobile Assistive Tool to Support } \\
\text { Children with Autism Practicing Social Skills in } \\
\text { Real-Life Situations [Escobedo 2012] }\end{array}$ & $\begin{array}{l}\text { Proceedings of the SIGCHI Conference on Human } \\
\text { Factors in Computing Systems (CHI '12) }\end{array}$ & 2012 \\
\hline $\begin{array}{l}\text { PIPLEX - Tangible Experience in an Augmented } \\
\text { Reality [Blanco 2010] }\end{array}$ & $\begin{array}{l}\text { Proceedings of the } 9^{\text {th }} \text { International Conference on } \\
\text { Interaction Design and Children (IDC '10) } \\
\end{array}$ & 2010 \\
\hline $\begin{array}{l}\text { Understanding and Designing Cool Technologies } \\
\text { for Teenagers [Kauppinen 2013] }\end{array}$ & $\begin{array}{l}\text { Proceedings of International Conference on Making } \\
\text { Sense of Converging Media (AcademicMindTrek } \\
\text { '13) }\end{array}$ & 2013 \\
\hline
\end{tabular}

Dentre os artigos listados se destaca, inicialmente, a questão dos jogos de tabuleiro. Os artigos de Mahmud et al. (2007) e de Marco et al. (2009) fazem uso desta abordagem, sendo que o primeiro constitui um estudo de caso que busca avaliar o potencial psicossocial da interação entre as crianças (inclusive, os autores indicam a necessidade de melhoria da forma de medir as médias). Destaca-se que a interface se apresenta de maneira a configurar a diversão das crianças-usuárias, propiciando uma comunicação e interação entre os participantes [Vygotsky 2001] por meio do uso de Visual Interaction Platform (VIP), tipo de Interface Natural (Natural User Interface NUI) que trabalha com as habilidades de manipulação de objetos para interação entre os usuários. De forma distinta, apesar de adotar o mesmo tipo de jogo (tabuleiro), Marco et al. (2009) desenvolveram uma mesa-tabuleiro que apresenta interação tangível para crianças do jardim da infância por meio de uma fazenda (sendo os bonecos e objetos virtuais). Com isso os autores objetivaram o desenvolvimento psicomotor de crianças de 3 a 4 anos. Em ambos os trabalhos a preocupação com ICC associada ao uso de RA como elemento que agregasse estímulos à cena se fez presente, instituindo possibilidades que contribuem para o desenvolvimento infantil por meio de uma interface amigável [Kirner et. al. 2006].

Outro aspecto a ser destacado, a partir da análise dos artigos que satisfaziam aos requisitos da revisão sistemática, diz respeito ao conceito de Interface com Usuário Tangível (IUT) [Zaman et al. 2012, Lagerstam, Olsson e Harviainen 2012, Blanco 2010]. Tais interfaces, de acordo com a abordagem de Zaman et al. (2012), buscam fornecer uma representação tangível para a informação digital e controles, propiciando 
ao usuário, literalmente, pegar/segurar os dados com as mãos e ter uma sensação de manipulação física desta representação. Cabe ressaltar também que os trabalhos atuais em IUT se voltam para aplicações destinadas às crianças, sendo que a relevância deste tipo de interface para a educação já constitui uma preocupação desde os trabalhos realizados por Papert (1993) com Mindstorms na década de 80. Destaca-se ainda que IUT envolvendo jogos educacionais infantis [Barbatsis et al. 2011] se caracterizam pelo foco no entretenimento, uso do potencial dos usuários, além da socialização e colaboração [Vygotsky 2001]. Em relação à usabilidade da interface, conforme Kirner et al. (2006), IUT trabalha com habilidades inatas dos usuários, apresentando benefícios potenciais para a aprendizagem de crianças já que vincula a manipulação concreta ao elemento cognitivo associado por meio de metáforas. Já Lagerstam, Olsson e Harviainen (2012) vinculam IUT ao uso de câmera de rastreamento de marcador de RA que projeta personagens em 3D. Assim, conforme o movimento da câmera o personagem também se move; em relação aos resultados este estudo trabalhou com crianças com experiência anterior com videogames, assim a manipulação da câmera não se tornou natural, causando estranheza às mesmas e tornando o aplicativo enfadonho. Finalmente, Blanco (2010) faz uso de IUT para apresentar um jogo em que os usuários devem criar um cenário por meio do uso de cartões coloridos de papelão, sendo que o personagem (constituído por um pinguim virtual) se movimenta pela cena elaborada. Com isso se buscou institui processos lúdicos e divertidos.

Já a proposta de Kauppinen (2013) faz uso de Kinect para propiciar a interação com um livro de histórias, sendo que o usuário seleciona o personagem que integrará a mesma. Esta investigação tinha como objetivo obter informações de como as crianças se comportariam ao utilizar Kinect para interagir e como os profissionais de educação poderiam vislumbrar uma forma mais ativa de interação e qual é o grau de complexidade desta para as crianças. Como resultados, os autores destacam que a interação gestual apresenta potencialidades para facilitar o processo de interação, mas também identificaram a necessidade de encontrar uma forma mais precisa de captura dos gestos.

Duas outras abordagens relacionam-se à língua estrangeira [McNally 2014] ou elementos culturais [Garzotto 2007]. McNally (2014) faz uso de tecnologia móvel para trabalhar a língua alemã com crianças, sendo que foram comparadas duas versões: a primeira requer a movimentação dinâmica do dispositivo e na segunda pauta-se na interação estática por meio de touchscreen. A análise dos resultados apontou que a segunda seria, num primeiro momento, mais aceita devido à facilidade, contudo posteriormente a primeira também foi utilizada. Numa abordagem distinta, Garzotto (2007) descreve um estudo de caso de um jogo multiusuário para web denominado de "O Tesouro dos Piratas", sendo que seu objetivo educacional voltou-se ao estímulo do interesse e atenção aos modos de vida pertinentes a outras culturas. A conclusão do estudo aponta a eficácia desta estratégia no processo educacional no que diz respeito à aprendizagem de novos conteúdos e á comunicação social.

Ainda deve-se destacar que um dos trabalhos [Magielse e Markopoulos 2009] era direcionado à atividade física (sendo que os batimentos cardíacos são monitorados pelo usuário, que é orientado ao longo da atividade com base nas informações coletadas), área contemplada por trabalhos que associam RA à Exergames [Maamar, Boukerche e Petriu 2012, Görgü et al. 2010a, Görgü et al. 2010b). 
Finalmente, Escobedo (2012) é a única referência que utiliza a abordagem estudada para crianças com autismo. Para isso faz uso de dispositivos móveis e RA como interface de aplicação, sendo que ao inicia-la são apresentadas opções para interação por meio da escolha do interlocutor para a comunicação entre dois estudantes.

\section{Considerações Finais}

Os resultados do estudo realizado possibilitaram identificar as áreas de aplicação dos estudos primários e averiguar que as mesmas destinam-se a processos educacionais (QP1). Assim, constitui uma lacuna na literatura a abordagem destinada somente ao entretenimento das crianças. Também se verificou que somente na base da ACM (QP2) foram encontrados artigos que tratavam dos três elementos. As demais bases não apresentavam trabalhos na área de ICC. Em relação ao histórico de combinação (QS1) de ICC, RA e jogos digitais, este remete a 2007, constituindo uma área de aplicação recente, sendo que o veículo de publicação (QS2) específico identificado é o International Conference on Interaction Design and Children da ACM.

Depois de verificar a problematização de se mesclar a RA a jogos e abordar uma interação motivadora para crianças, se pretende, como trabalho futuro, averiguar uma plataforma de software que permita oferecer infraestrutura a estas possibilidades.

\section{Agradecimentos}

Os autores agradecem à Universidade Presbiteriana Mackenzie e ao MackPesquisa pelo suporte disponibilizado para a realização da investigação.

\section{Referências}

Barbatsis, K., Economou, D., Papamagkana, I. e Loukas, D. (2001). 3D environments with games characteristics for teaching history: the VRLerna case study. In Proceedings of the $29^{\text {th }}$ ACM international conference on Design of communication (SIGDOC '11). ACM, New York, NY, USA, 59-66.

Blanco J. M., Landry P., Mealla S. C., Mazzone E. e Parés N. (2010). PIPLEX: tangible experience in an augmented reality video game. In Proceedings of the $9^{\text {th }}$ International Conference on Interaction Design and Children (IDC '10). ACM, New York, NY, USA, 274-277.

Escobedo L., Nguyen D. H., Boyd L., Hirano S., Rangel A., Garcia-Rosas D.1, Tentori M. e Hayes G.( 2012). MOSOCO: a mobile assistive tool to support children with autism practicing social skills in real-life situations. In Proceedings of the SIGCHI Conference on Human Factors in Computing Systems (CHI '12). ACM, New York, NY, USA, 2589-2598.

Garzotto F. (2007). Investigating the educational effectiveness of multiplayer online games for children. In Proceedings of the $6^{\text {th }}$ international conference on Interaction design and children (IDC '07). ACM, New York, NY, USA, 29-36.

Görgü, L., Campbell, A. G., McCusker, K., Dragone, M., O'Grady, M. J., O'Connor, N.E. e O'Hare, G. M. P. (2010). FreeGaming: mobile, collaborative, adaptive and augmented exergaming. In Proceedings of the 8th International Conference on 
Advances in Mobile Computing and Multimedia (MoMM '10). ACM, New York, NY, USA, 173-179.

Gorgu, L., Campbell, A. G., Dragone, M., e O'Hare, G. M. P. (2010) ACM Computers in Entertainment (CIE) Magazine, 8 (4): Article No. 27, 4p.

Harada, Y., Nosu, K. e Okude, N. (1999). Interactive and Collaborative Learning Environment using 3D Virtual Reality Content, Multi-Screen Display and PCs. In Proceedings of the $8^{\text {th }}$ Workshop on Enabling Technologies on Infrastructure for Collaborative Enterprises (WETICE '99). IEEE Computer Society, Washington, DC, USA, 238-244.

Kauppinen S., Luojus S., Tuomisto J. e Ahlgren A. (2013). Utilizing Gesture Recognition Technology in Children's Interactive Storybook. In Proceedings of International Conference on Making Sense of Converging Media (AcademicMindTrek '13), Artur Lugmayr, Heljä Franssila, Janne Paavilainen, and Hannu Kärkkäinen (Eds.). ACM, New York, NY, USA, Pages 76, 4 p.

Kirner, C., Zorzal, E. R., Kirner, T. G. Case Studies on the Development of Games using augmented Reality. In: Proceedings of 2006 IEEE International Conference on Systems, Man, and Cybernetics, 2006, Taipei, v. 1. p. 14-19.

Lagerstam E., Olsson T. e Harviainen T. (2012). Children and intuitiveness of interaction: a study on gesture-based interaction with augmented reality. In Proceeding of the $16^{\text {th }}$ International Academic MindTrek Conference (MindTrek '12). ACM, New York, NY, USA, 169-174.

Maamar H. R., Boukerche A. e Petriu, E. M. (2012). 3-D Streaming Supplying Partner Protocols for Mobile Collaborative Exergaming for Health. In IEEE Transactions on Information Technology in Biomedicine, 16(6):1079-1095.

Mahmud A. A., Mubin O., Octavia J. R., Shahid S., Yeo L., Markopoulos P. e Martens J. (2007). aMAZEd: designing an affective social game for children. In Proceedings of the $6^{\text {th }}$ international conference on Interaction design and children (IDC '07). ACM, New York, NY, USA, 53-56.

Magielse R. e Markopoulos P. (2009). HeartBeat: an outdoor pervasive game for children. In Proceedings of the SIGCHI Conference on Human Factors in Computing Systems (CHI '09). ACM, New York, NY, USA, 2181-2184.

Marco J., Cerezo E., Baldassarri S., Mazzone E. e Read J. C. (2009). Bringing tabletop technologies to kindergarten children. In Proceedings of the 23rd British HCI Group Annual Conference on People and Computers: Celebrating People and Technology (BCS-HCI '09). British Computer Society, Swinton, UK, UK, 103-111.

McNally B., Guha M. L., Norooz L., Rhodes E. e Findlater L. (2014). Incorporating peephole interactions into children's second language learning activities on mobile devices. In Proceedings of the 2014 conference on Interaction design and children (IDC '14). ACM, New York, NY, USA, 115-124.

Papert, S. Mindstorms: Children, Computers, and Powerful Ideas. Basic Books, 1993.

Papert, S. A máquina das crianças: repensando a escola na era da informática. Ed. Ver. Porto Alegre: Artmed. 2008. 
Read, J.C. e Bekker, M.M. (2011) The Nature of Child Computer Interaction. In Proceedings of BCS-HCI 2011, Northumbria, UK.

Vygotsky, L. S. (2001). A construção do pensamento e da linguagem. São Paulo, Martins Fontes.

Tori, R., Kirner, C. e Siscoutto, R. A. Fundamentos e tecnologia de realidade virtual e aumentada. Editora SBC, 2006.

Zaman B., Abeele V. V., Markopoulos P. e Marshall P. (2012). Editorial: the evolving field of tangible interaction for children: the challenge of empirical validation. Personal Ubiquitous Comput. 16, 4 (April 2012), 367-378. 